\title{
铜催化嗍试剂参与的烯烃多组分双官能化研究进展
}

\author{
杨 晨 贾雪锋* \\ (山西师范大学化学与材料科学学院 磁性分子与磁信息材料教育部重点实验室 山西临汾 041000)
}

\begin{abstract}
摘要 烯烃的多组分双官能化是一类高效快速构建结构多样性分子的重要有机反应，具有原子经济性高、底物范围广 及条件温和等诸多优点, 近年来成为有机合成领域的主要研究热点. 许多金属或非金属催化条件下的烯烃多组分双官 能化反应已经被大量报道, 借助手性配体, 有的反应还成功地实现了不对称双官能化. 然而, 与贵重金属催化剂(钉、 铑、铱、钯、银)相比, 价格低廉的过渡金属(铁、钴、镍、铜、锰)催化的有机反应更加受到人们的青睐. 考虑到铜催 化剂和有机嗍试剂在有机合成中的应用, 针对近三年来铜催化下有机嗍试剂参与的烯烃多组分双官能化反应进行了综 述, 并对该领域面临的挑战和发展方向进行了展望.
\end{abstract}

关键词 铜催化; 嗍试剂; 烯烃; 多组分双官能化

\section{Recent Advances in Copper-Catalyzed Boron-Reagent-Involved Multicomponent Difunctionalization of Alkenes}

\author{
Yang, Chen Jia, Xuefeng* \\ (Key Laboratory of Magnetic Molecules \& Magnetic Information Materials (Ministry of Education), School of Chemistry and \\ Material Science, Shanxi Normal University, Linfen, Shanxi 041000)
}

\begin{abstract}
The multicomponent difunctionalization of alkenes is an important and highly efficient reaction for the rapid construction of molecules with structure diversity, which has some advantanges of high atom economy, wide substrate range as well as mild conditions and becomes the main research hotspot in organic synthesis in recent years. Many examples on the multicomponent difunctionalization of alkenes under metal-catalyzed or metal-free conditions have extentively been reported. Among them, the asymmetric version of some reactions was successfully achieved by the use of chiral ligands. However, compared with nobel metal catalysts such as ruthenium, rhodium, iridium, palladium and silver, the cheaper metal catalysts including iron, cobalt, nickel, copper, manganese have attracted more special attention. In view of the application of copper catalyst and organoboron reagents in organic synthesis, the advances in copper-catalyzed multicomponent difunctionalization of alkenes with organoboron reagents since 2018 are reviewed. The major challenges and development prospects of this fields, including the discovery of new reaction type and organoboron reagents, better stereoselectivity, and deeper understanding on reaction mechanism are also discussed.
\end{abstract}

Keywords copper-catalysis; boron reagent; alkene; multicomponent difunctionalization

烯烃的双官能化反应可以在双键碳原子上同时引 入两个官能团，具有经济、高效、底物适用广以及条件 温和等诸多优点, 为结构多样性有机化合物的合成提供 了一种新策略. 目前烯烃的双官能化反应仍然是有机合 成领域的重要研究热点, 吸引着众多合成化学家去发现 新的催化体系和反应. 2017 年张万斌课题组 ${ }^{[1]}$ 综述了金 属催化下共轭二烯的 1,2-双官能化反应的研究进展. 2019 年, 赵文献课题组 ${ }^{2]}$ 对过渡金属(包括铜、铁、锰、
铑、铱、银、钯等金属)和无金属催化下烯烃的多种双 官能化反应进行了系统的总结, 使我们对该领域的研究 进展有了更为深刻的全面认识. 随后, 余达刚课题组 ${ }^{[3]}$ 重点阐述了二氧化碳参与的自由基型烯烃的四类双官 能化反应研究进展及机理. 另一方面, 过渡金属催化的 多组分反应通过使用三个或三个以上的底物可以一步 高效地构建单一产物分子, 具有很高的原子经济性和合 成效率，是快速合成多官能团复杂有机分子的有效手

* Corresponding author. E-mail: jxfliu@163.com

Received December 4, 2020; revised January 18, 2021; published online January 28, 2021.

Project supported by the Natural Science Foundation of Shanxi Province (No. 201901D111276).

山西省自然科学基金(No. 201901D111276)资助项目. 
段. 2020 年 Engle 课题组 ${ }^{[4]}$ 总结了镍催化下各种不同类 型烯烃的多组分 1,2-双碳官能化研究的最新进展. Molander 课题组 ${ }^{[5]}$ 对光氧化还原/镍协同催化烯烃的 1,2-双 官能化多组分反应进行了归纳, 指出当前研究面临的挑 战. 江焕峰课题组 ${ }^{[6]}$ 综述了偕二氟代烯烃的三组分官能 化反应，这些反应对于含氟有机化合物的合成具有很好 的参考价值. 与贵金属催化剂相比, 铜化合物作为一类 来源丰富、价格便宜且低毒性的金属催化剂, 具有得天 独厚的优势，在过渡金属催化的有机反应中应用较广， 近年来在烯烃的双官能化反应研究中的应用尤为明

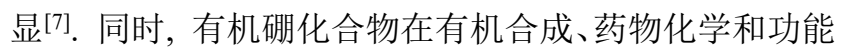
材料领域应用广泛, 发展新型高效的含硼化合物的合成 方法一直都是有机合成领域的研究热点. 根据反应中有 机嗍试剂的不同, 铜催化下烯烃的多组分双官能化主要 通过两种反应模式进行: (a)在存在配体、碱和铜盐的情 况下, 首先产生一个活性的 $\mathrm{L}_{n} \mathrm{CuX}$ 物种, 与联硼酸频哪 醇酯 $\left(\mathrm{B}_{2} \mathrm{pin}_{2}\right)$ 作用生成硼基酮化合物 $\mathrm{L}_{n} \mathrm{CuBpin}$, 接着与 烯烃加成获得亲核性 $\beta$-嗍烷基铜配合物中间体，该中间 体与亲电体底物反应经还原消除后提供双官能化产物, 并再生活性的 $\mathrm{L}_{n} \mathrm{CuX}$; (b) 在存在过氧化物或光催化剂条 件下, 反应经单电子转移(SET)生成一个活性的自由基
$\mathrm{R} \cdot$ ，再与烯烃分子加成形成新的自由基，该自由基会和 体系中芳基硼酸与铜盐、配体生成的芳基铜配合物 $\mathrm{ArCu}^{\mathrm{II} L}$ 反应，生成的三价铜中间产物经还原消除后得 到目标产物, 或者该自由基直接与 $\mathrm{ArCu}^{\mathrm{II}} \mathrm{L}$ 经芳基迁移 过程转化为产物, 并再生 $\mathrm{LCu}^{\mathrm{I} X}$ 催化剂(Scheme 1). 本 文对近三年来铜催化下有机嗍试剂参与的烯烃多组分 双官能化反应进行了总结, 并对该领域的发展作一展 望.

\section{$1 \mathrm{~B}_{2} \mathrm{pin}_{2}$ 参与的烯烃多组分双官能化反应}

\section{1 烯烃的硼烷基化反应}

2018 年, Kana 课题组 ${ }^{[8]}$ 报道了铜催化下苯乙烯、 $\mathrm{B}_{2} \mathrm{pin}_{2}$ 与亚胺的对映和非对映立体发散性三组分硼化偶 联反应，以高的产率获得了对映体富集的 $\alpha, \beta$-双分支 $\gamma$ 硼基胺衍生物(Eq. 1). 通过选择恰当的手性配体和铜催 化剂, 反应可选择性地获得产物分子的任何一个立体异 构体. 该方法结合了催化剂控制的立体发散性和 $\mathrm{B}_{2} \mathrm{pin}_{2}$ 的反应性, 为分子结构多样性的构筑并精准控制分子的 手性提供了新的策略.

(a) Borofunctionalization of alkenes with $\mathrm{B}_{2} \mathrm{pin}_{2}$ via nucleophilic $\beta$-borylalkyl copper complex

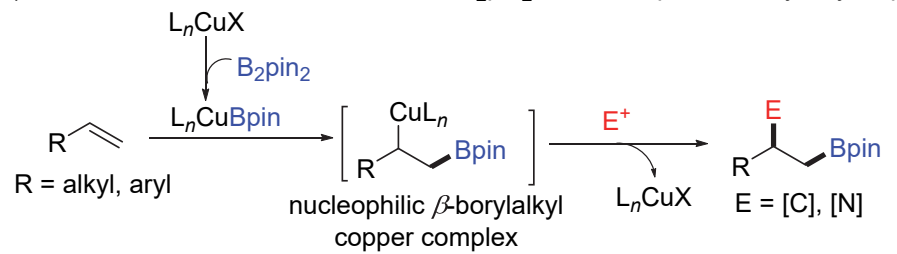

(b) Difunctionalization of alkenes with $\mathrm{ArB}(\mathrm{OH})_{2}$ by radical relay coupling strategy

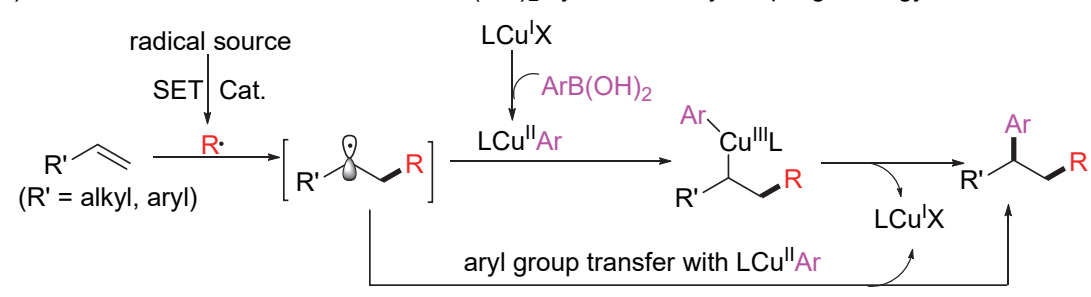

图式 1 铜催化嗍试剂参与的烯烃多组分双官能化反应模式

Scheme 1 Reaction pattern on copper-catalyzed multicomponent difunctionalization of alkenes with organoboron reagents
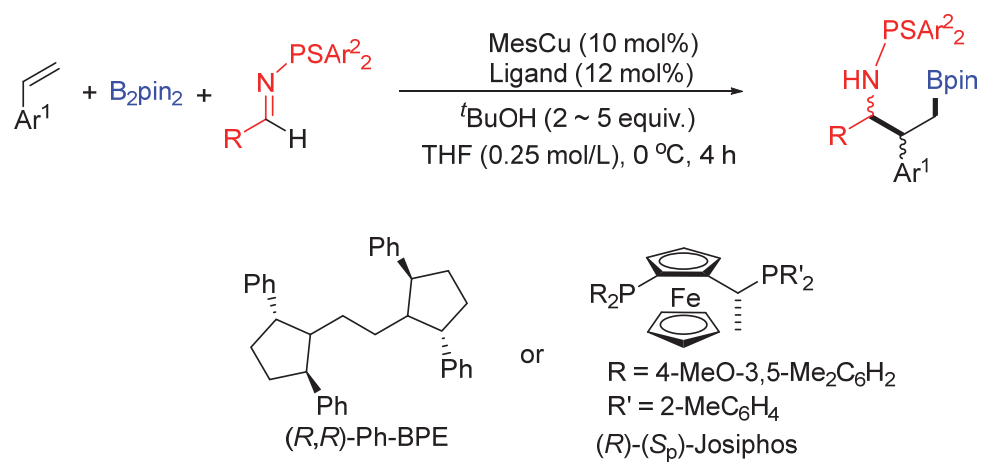
同年, Hoveyda 课题组 ${ }^{[9]}$ 发展了铜和手性双膦配体 催化芳基烯烃的对映选择性硼烷基化反应(Eq. 2). 作者 通过实验和理论计算详细研究了多种因素如烯烃的电 子特性、反应物间的比例、亲电体大小和浓度等对反应 对映选择性的影响, 揭示了反应的活性物种 LCuBpin 在 催化循环过程中的关键作用.

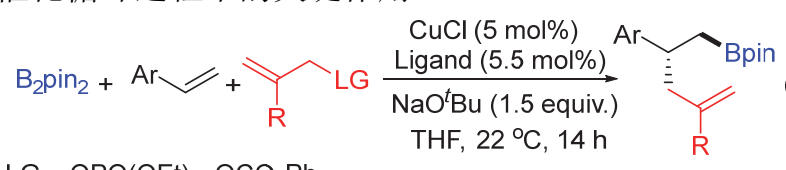

$\mathrm{LG}=\mathrm{OPO}(\mathrm{OEt})_{2}, \mathrm{OCO}_{2} \mathrm{Ph}$

$$
\text { Ar', } \mathrm{Ar} "=\mathrm{Ph} ; \mathrm{Ar}^{\prime}=\mathrm{Ph}, \mathrm{Ar}^{\prime \prime}=3,5-\mathrm{Me}_{2} \mathrm{C}_{6} \mathrm{H}_{3}
$$

\section{2 烯烃的嗍氨基化反应}

2018 年, 王剑波等 ${ }^{[10]}$ 发展了一种铜催化下苯乙烯 的区域选择性氨基嗍化反应(Eq. 3). 在 $\mathrm{Cu}(\mathrm{OAc})_{2}$ 为催化 剂, 1,2-双 (二苯基膦)苯( $\mathrm{dppbz}$ )为配体, $\mathrm{KO} \mathrm{O}^{t} \mathrm{Bu}$ 为碱, 1,4二氧六环为溶剂的条件下, 利用 $\mathrm{B}_{2} \mathrm{pin}_{2}$ 为亲核性的嗍源, 偶氮化合物为亲电胺化试剂, 以良好的产率获得一系列 硼化的腙类化合物. 反应中原位生成的茮基铜物种被偶 氮化合物中亲电性的氮捕获, 以区域选择性的方式获得 氨基嗍化产物。

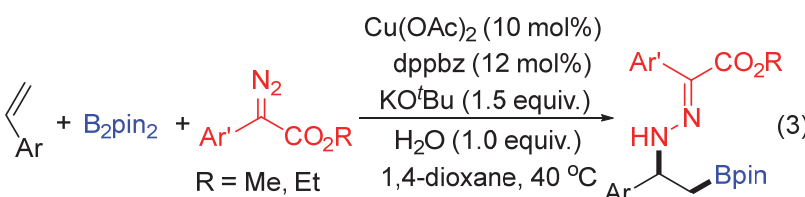

$$
\begin{aligned}
& \text { dppbz: }
\end{aligned}
$$

同年, Miura 课题组 ${ }^{[11]}$ 报道了 $\mathrm{CuCl} /$ 手性双膦配体催 化未活化烯烃的不对称硼氨基化反应，使用 $\mathrm{B}_{2} \mathrm{pin}_{2}$ 和羟 胺类化合物作为官能化试剂, 以良好的产率获得手性 $\beta$ 嗍基胺(Scheme 2). 由于该产物不稳定, 不能使用柱色 谱进行分离纯化, 所以需要将其氧化为手性氨基醇, 再 确定反应的对映选择性. 该反应主要适用于烷基和环烷 基取代的烯烃分子以及含不同取代基羟胺化合物, 其官 能化产物在药物化学中有着潜在的应用价值.

2019 年, Zhang 课题组 ${ }^{[2]}$ 报道了一例铜催化芳基烯 烃的不对称氨基硼化反应(Eq. 4). 反应以 $\mathrm{CuOTf}$ 为催化 剂, ${ }^{P i}$ ZPhos 为手性配体, 芳基烯烃、 $\mathrm{B}_{2} \mathrm{pin}_{2}$ 和 $O$-苯甲酰 基- $N, N$-二芐基羟胺 $\left(\mathrm{Bn}_{2}-\mathrm{N}-\mathrm{OBz}\right)$ 为底物, 表现出良好的 对映选择性, 室温下获得的硼氨基化产物经四水合硼酸 钠 $\left(\mathrm{NaBO}_{3} \cdot 4 \mathrm{H}_{2} \mathrm{O}\right)$ 氧化后, 转化为手性氨基醇. 该方法可
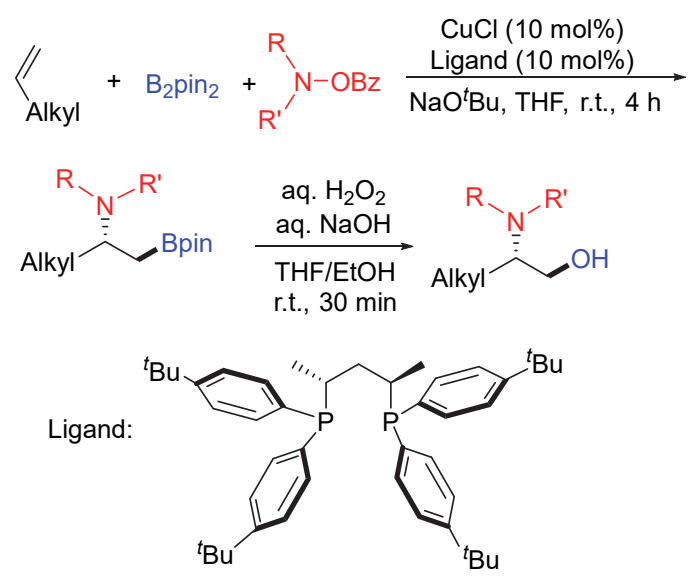

图式 2 铜催化烷基烯烃的不对称嗍氨基化反应

Scheme 2 Copper-catalyzed asymmetric aminoboration of alkyl-substituted alkenes

以进一步扩展到杂芳基烯烃如 3-吡啶基乙烯, 获得了优 异的对映选择性.

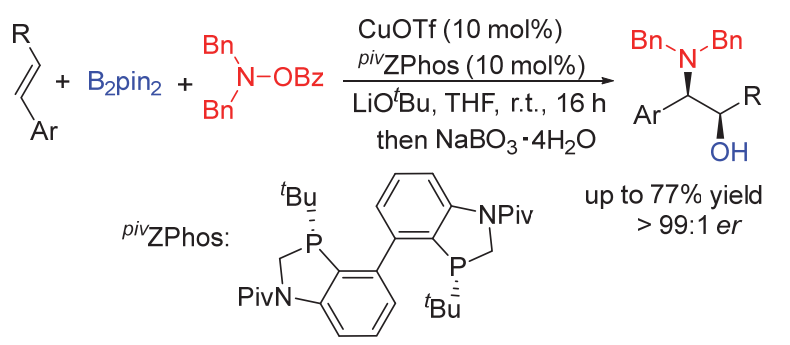

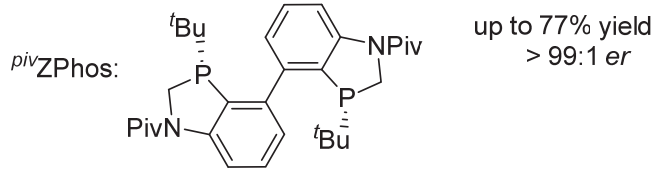

\section{3 烯烃的硼芳基化反应}

2019 年, Brown 课题组 ${ }^{[13]}$ 利用 $\mathrm{B}_{2} \mathrm{pin}_{2}$ 、芳基溴化物 为官能化试剂, 发展了催化剂控制的 $\alpha$-烷基烯基芳烃的 1,2-和 1,1-芳基硼化反应(Scheme 3). 两种官能化反应均 在 $\mathrm{Cu}-\mathrm{Pd}$ 双催化体系下进行, 使用相同的铜催化剂 (SIMesCuCl), 通过两种不同钯催化剂( $\mathrm{APhosPdG}_{3}$ 和 $\left.\mathrm{PCy}_{3} \mathrm{PdG}_{3}\right)$ 的调控作用, 经由一个相同的中间体, 采取 不同的反应途径转化为相应的官能化产物. 机理的研究 表明，在 1,2-芳基硼化反应中，反应通过很少见的三级 烷基金属有机配合物的偶联形成季碳中心; 而在 1,1-芳 基嗍化中，反应更倾向于按照 $\beta$ - $\mathrm{H}$ 消除/迁移插入的方 式转化为 1,1-官能化产物. 值得一提的是, 如果使用手 性的氮杂环卡宾配合物作为催化剂, 这两个反应均可以 实现 $\alpha$-烷基烯基芳烃的不对称 1,2-和 1,1-芳基硼化反应.

2020 年, Yun 课题组 ${ }^{[14]}$ 报道了钯和铜协同催化下烯 基硼化合物(alkenyl-Bdan)的不对称芳基硼化反应 (Scheme 4). 反应中利用噻吩-2-甲酸亚铜(CuTC)和手性 氮杂环卡宾配体, 通过 NHC-Cu-Bpin 物种对烯基硼化 合物的加成, 产生一个对映体富集的 $\alpha$-硼化有机铜活性 中间体，随后在 Pd-XPhos 催化剂作用下与芳基澳化物 进行立体专一性的交叉偶联反应，最终以良好的产率和 


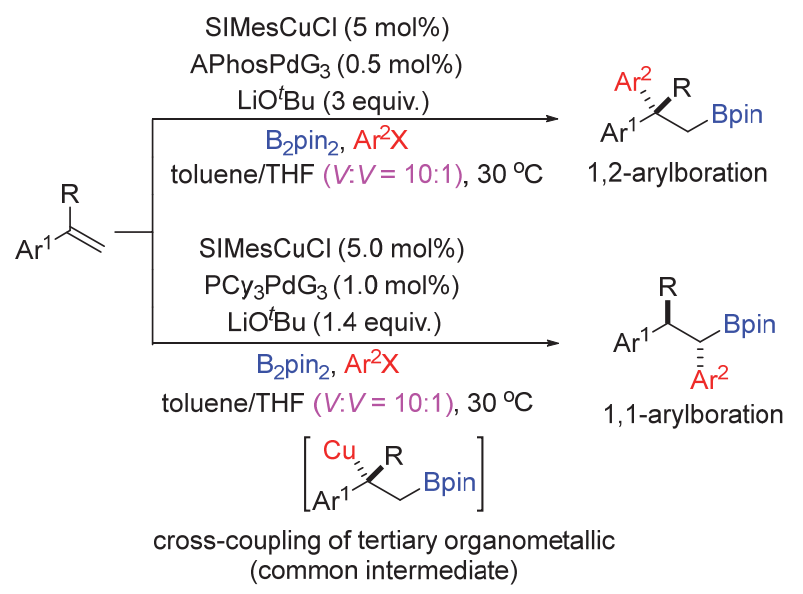

图式 3 催化剂控制的 $\alpha$-烷基烯基芳烃的 1,1-和 1,2-芳基嗍化 反应

Scheme 3 Catalyst-controlled 1,1- and 1,2-arylboration of $\alpha$-alkyl alkenyl arenes

高的对映选择性得到结构多样化的 1,2-二硼基取代的烷 烃衍生物, 该产物经氧化处理后可以转化为反式-1,2-二 羟基化合物. 该方法的成功之处是在烯烃的 $\alpha$-碳原子上 引入了一个 1,8 -䒺二胺硼基(Bdan)取代基, 同时双齿的 手性咪唑盐配体与铜催化剂有效地产生了对映体富集 的 $\alpha$-嗍化有机铜中间体, 促使反应以立体专一性的方式 与芳基溴化物发生偶联反应.

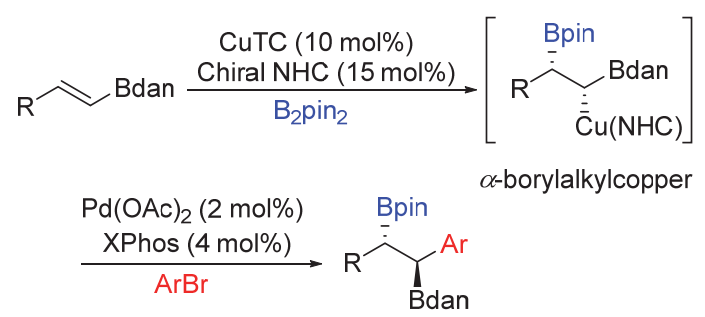

up to $>99:<1 d r$ up to $96.5: 3.5 \mathrm{er}$

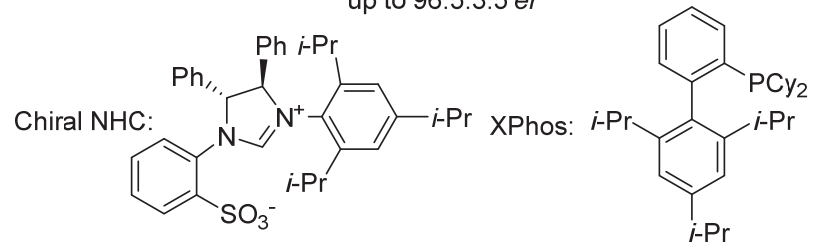

图式 4 铜和钯共催化烯基硼化合物的立体专一性芳基硼化 反应

Scheme 4 Copper- and palladium-cocatalyzed stereospecific arylboration of alkenyl-Bdan compounds

2020 年, 刘元红课题组 ${ }^{[15]}$ 发展了铜催化苯乙烯的 杂芳基嗍化反应，使用 $N$-氧化喹啉作为杂芳基化试剂, $\mathrm{B}_{2} \mathrm{pin}_{2}$ 为喼化试剂, 获得了多种 $\mathrm{C}(2)$-官能化的喹啉衍生 物, 有望应用在药物化学和有机合成领域(Scheme 5). 在催化循环中，烷氧基铜配合物与 $\mathrm{B}_{2} \mathrm{pin}_{2}$ 作用生成 $\mathrm{LCu}(\mathrm{I})-\mathrm{Bpin}$ 的活性物种与苯乙烯生成 $\beta$-碀基烷基铜中

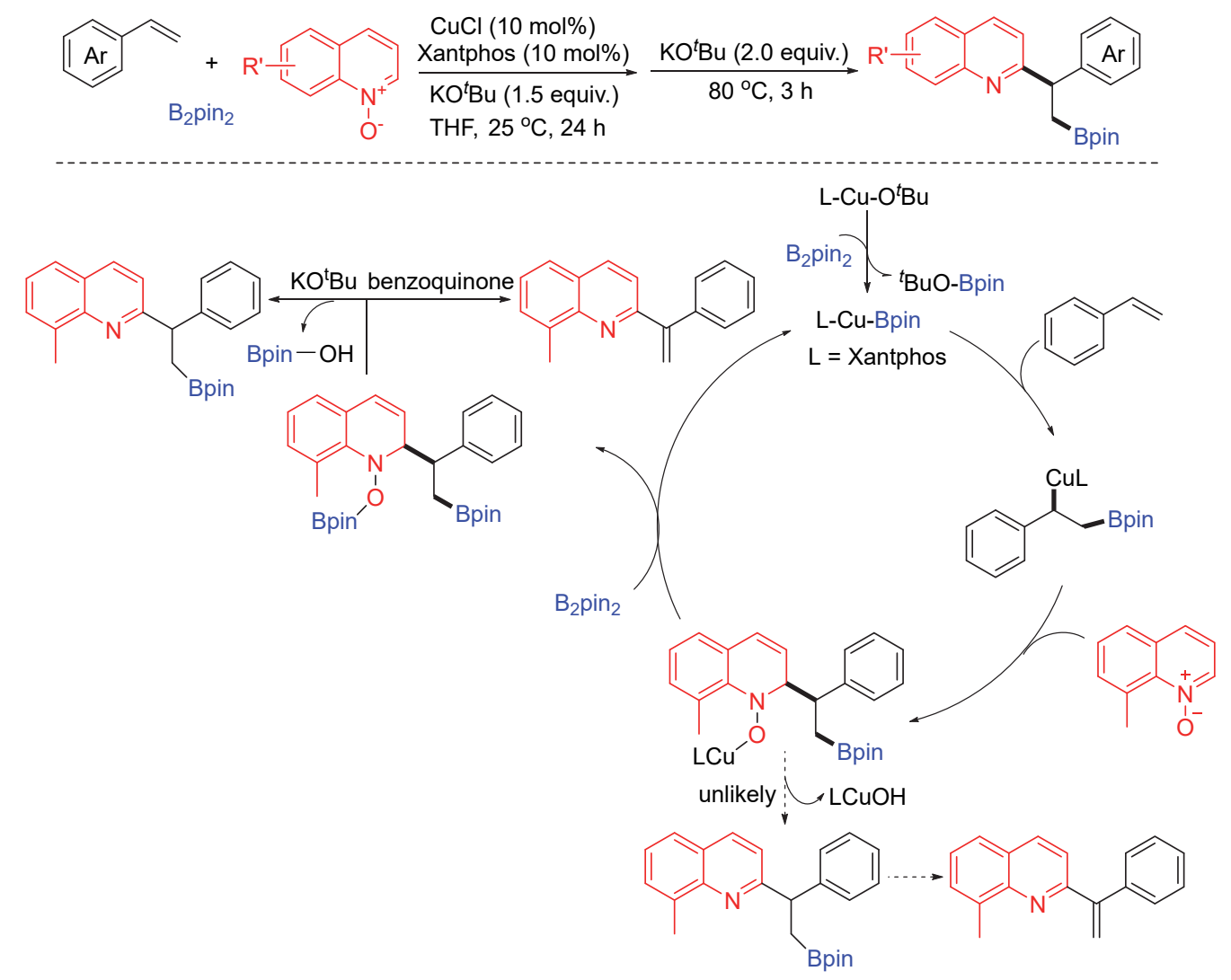

图式 5 铜催化芳基烯烃的杂芳基嗍化反应及可能的催化循环

Scheme 5 Copper-catalyzed borylative heteroarylation of vinylarenes and proposed catalytic cycle 
间体, 其亲核进攻 $N$-氧化喹啉的 $\mathrm{C}(2)$ 得到加成产物, 再 依次经 $\mathrm{B}_{2} \mathrm{pin}_{2}$ 、苯醌或 $\mathrm{KO}^{t} \mathrm{Bu}$ 处理后转化为目标分子. 苯醌或 $\mathrm{KO}{ }^{t} \mathrm{Bu}$ 对产物分子的形成起至关重要的作用, 当 反应体系中存在苯醌时, 中间体会转化为 $\mathrm{C}(2)$-烯基化 的喹啉分子, 而在无氧化剂条件下, 使用 $\mathrm{KO}^{t} \mathrm{Bu}$ 处理, 则得到预期的双官能化产物(Scheme 5).

\section{4 烯烃的磞羧基化反应}

2016 年, Popp 课题组 ${ }^{[16]}$ 实现了铜卡宾 $[\mathrm{ICyCuCl}]$ 催 化二氧化碳参与的芳基烯烃和 $\mathrm{B}_{2} \mathrm{pin}_{2}$ 的区域选择性硼羧 基化反应(Eq. 5), 以中等到良好的产率获得了单一异构 体, 产物进一步可转化为在药物合成中具有较高应用价 值的化合物分子. 2019年, Lin 课题组 ${ }^{[17]}$ 利用密度泛函理 论(DFT)计算的方法对上述铜催化烯烃的三组分喼羧基 化反应机理进行了研究, 提出了反应的催化循环过程, 对反应涉及到的活性中间体、碱的作用及可能的竞争反 应进行了详细分析, 该研究成果成功地应用于不同硼试 剂参与的多组分反应可能性的预测.

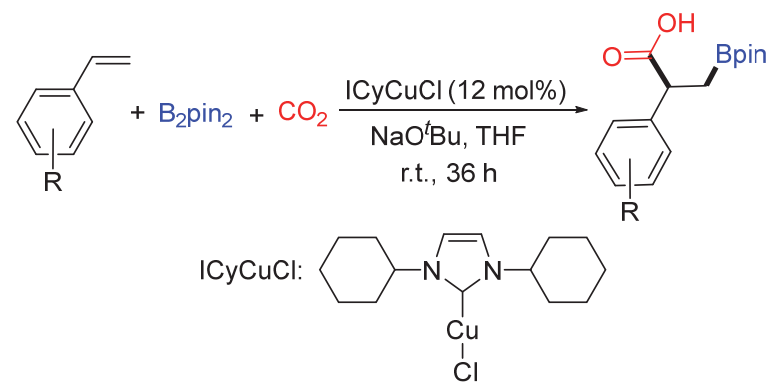

\section{5 烯烃的硼酰基化反应}

2017 年, Brown 课题组 ${ }^{[18]}$ 报道了铜催化下活泼烯烃 的嗍酰基化反应, 使用含不同取代基的酰氯和 $\mathrm{B}_{2} \mathrm{pin}_{2}$ 作 为官能化试剂. 对于大多数活泼烯烃底物, 反应只需要 较低的催化剂量(1 mol\%)和很短的时间(15 min)室温下 即可完成(Eq. 6). 反应同样适用于二烯烃和刚性的二环 烯烃. 如果使用手性的铜催化剂, 芳基烯烃还能实现不 对称硼酰基化反应, 以良好的对映选择性获得手性双官 能化产物.

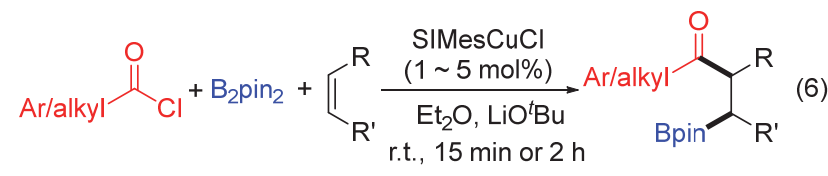

\section{6 烯烃的嗍酰胺化反应}

2020 年, Mazet 课题组 ${ }^{[19]}$ 利用空气稳定的卡宾铜 [(SIMes) $\mathrm{CuCl}]$ 催化剂, 发展了苯乙烯、芳基异氰酸酯和 $\mathrm{B}_{2} \mathrm{pin}_{2}$ 的三组分嗍酰胺化反应, 以良好的产率获得硼化
的二级酰胺类化合物. 反应具有良好的官能团兼容性, 底物范围广，可以扩展到炔烃、1,3-二烯及二环烯烃等. 进一步的研究表明, 通过引入一个手性的 P,N-配体, 可 以实现该反应的不对称诱导，以前所未有的对映选择性 获得一系列 $\alpha$-手性酰胺衍生物(Eq. 7). 反应物的电子特 性与产物的对映体比率之间的 Hammett 相关性表明，异 氰酸酯结构的变化比苯乙烯类底物更容易影响到反应 的对映选择性.

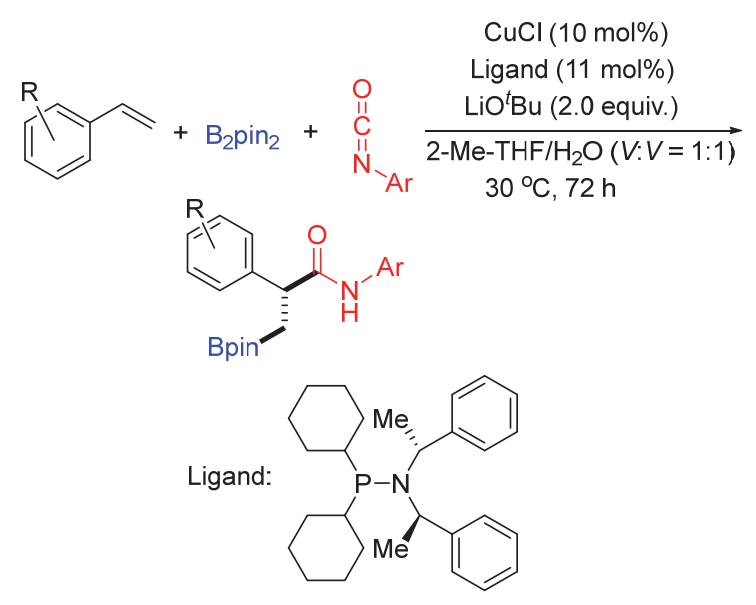

随后，陶传洲课题组 ${ }^{[20]}$ 也报道了一个铜催化下芳 基烯烃、异氧酸酯和 $\mathrm{B}_{2} \mathrm{pin}_{2}$ 的三组分硼酰胺化反应，该 反应适用于各种取代基的芳香烯烃和异氰酸酯底物，可 以良好的产率制备一系列含不同官能团的 $\beta$-硼化酰胺 (Eq. 8). 产物分子含有酰胺和硼酸酯基团，可通过后期 衍生化应用于药物分子和天然产物的合成.

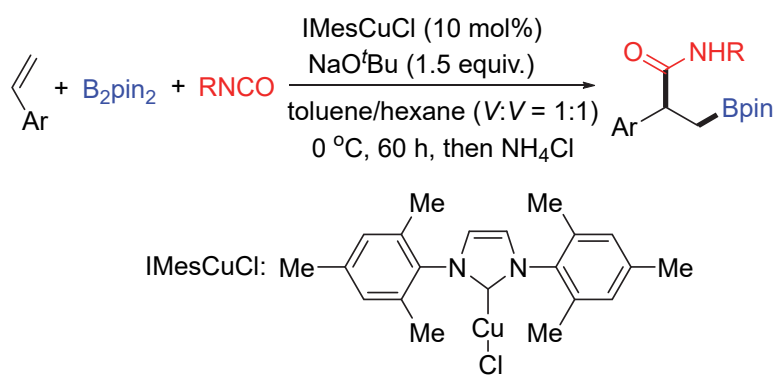

\section{7 烯烃的嗍羰基化反应}

2020 年, Wu 课题组 ${ }^{[21]}$ 发展了铜催化的未活化烯烃、 烷基卤代烃、一氧化碳 $(\mathrm{CO})$ 和 $\mathrm{B}_{2} \mathrm{pin}_{2}$ 的四组分偶联反应, 借助 $\mathrm{IPrCuCl} / \mathrm{Xantphos}$ 催化体系, 实现了烯烃的区域选 择性硼羰基化反应(Eq. 9). 反应条件温和，适用于烷基 烯烃、芳基烯烃和不同的烷基碘代烃，为 $\beta$-硼基酮衍生 物的合成提供了一种新的方法.

同年, $\mathrm{Wu}$ 课题组 ${ }^{[2]}$ 再次报道了 $\mathrm{Cu} / \mathrm{Pd}$ 双金属协同 催化下芳烯、芳基卤化物/三氟磺酸酯、 $\mathrm{B}_{2} \mathrm{pin}_{2}$ 和 $\mathrm{CO}$ 的 四组分硼羰基化反应(Scheme 6). 在氮杂环卡宾铜 


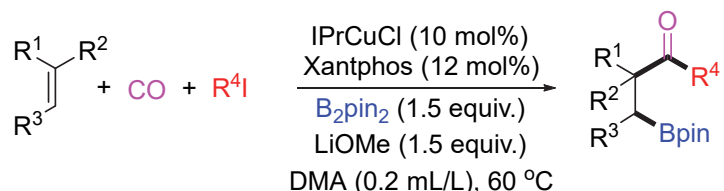
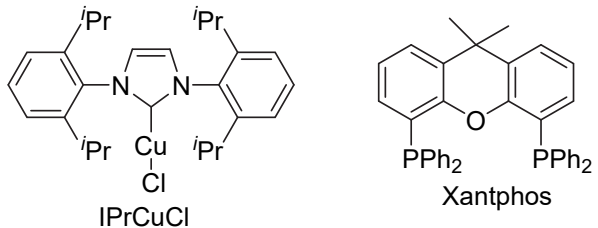

$\left(\mathrm{IMesCuCl}\right.$ 或 $\left.{ }^{M e} \mathrm{PrCuCl}\right)$ 、氯化烯丙基钯二聚物 $\left[\left(\eta^{3}-\mathrm{C}_{3} \mathrm{H}_{5}-\mathrm{PdCl}\right)_{2}\right]$ 和含磷配体 (dppp)的共同作用下, 以芳
基碘为底物，反应可以高产率地得到具有合成价值的 $\beta$ 硼基酮化合物; 以芳基三氟磺酸酯为底物，则可以得到 一系列兼容多种官能团的 $\beta$-硼基乙烯基酯衍生物. 机理 的研究表明, $\mathrm{NHC}-\mathrm{Cu} 、\left(\eta^{3}-\mathrm{C}_{3} \mathrm{H}_{5}-\mathrm{PdCl}\right)_{2}$ 和含磷配体 (dppp)对反应的发生起关键作用, 在铜、钯双催化循环 体系中，铜催化剂活化芳基烯烃，产生烷基铜中间体 I, $\mathrm{CO}$ 插入到烷基铜中间体 I 后转化为酰基铜中间体 II, II 互变异构转化为乙烯基烷氧铜化合物 III, 随后与芳基 碘/三氟磺酸酯、 $\mathrm{CO}$ 和钯催化剂形成的芳羰基钯中间体 IV 发生转金属化反应, $\mathrm{V}$ 经还原消除后得到 $\beta$-嗍基乙烯 基酯(Scheme 7).

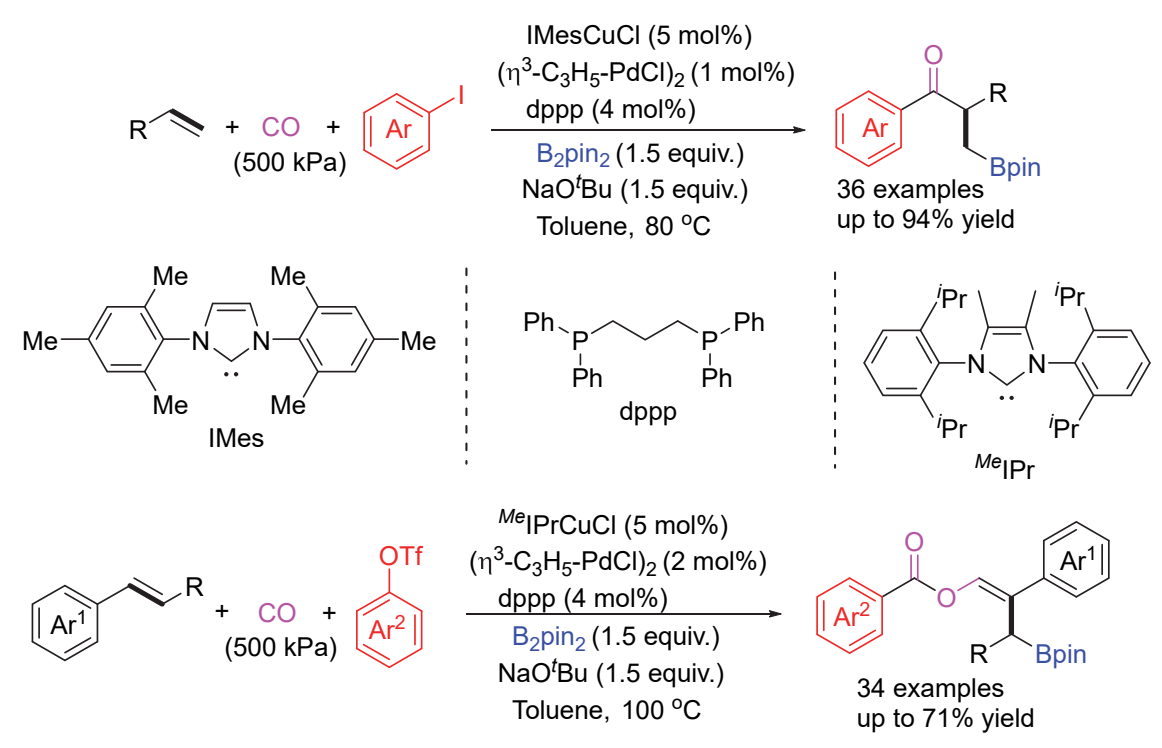

图式 6 铜和钯协同催化芳烯的四组分嗍羰基化反应

Scheme 6 Copper and palladium cooperative catalyzed four-component borocarbonylation of vinylarenes

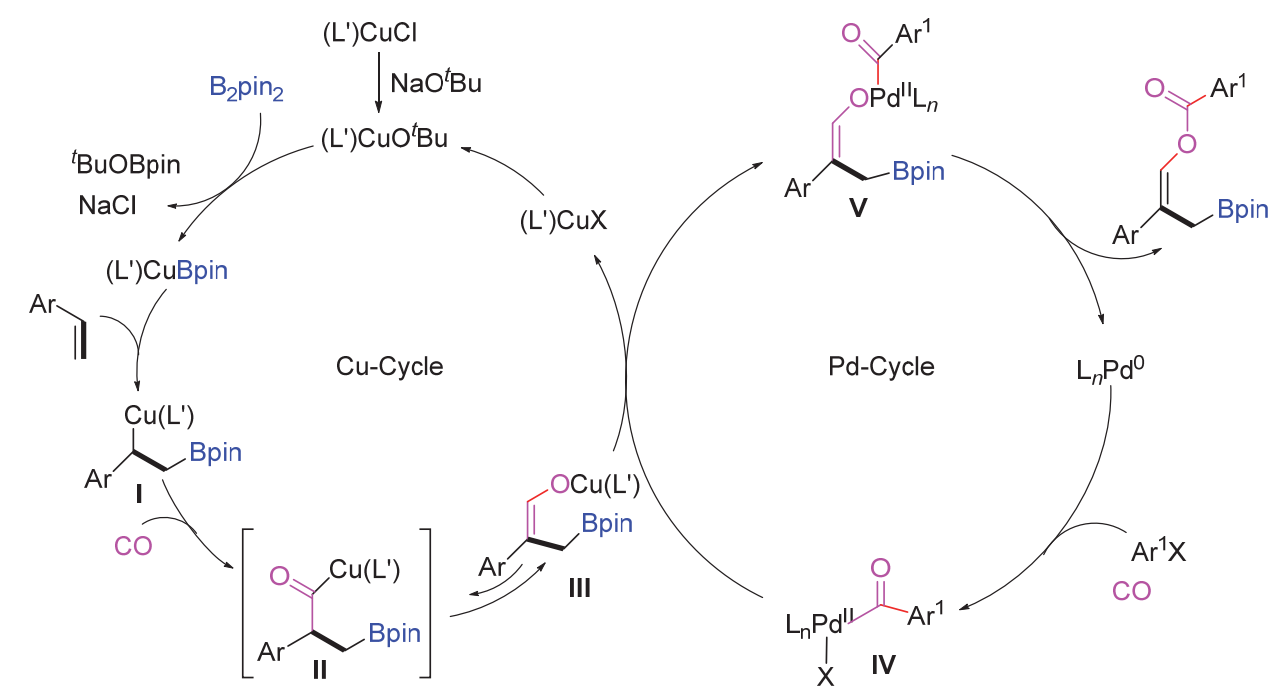

图式 7 铜和钯协同催化芳烯的四组分嗍羰基化反应机理

Scheme 7 Mechanism of copper and palladium cooperative catalyzed four-component borocarbonylation of vinylarenes 


\section{8 烯烃的环丙烷化反应}

2020 年, $\mathrm{Wu}$ 课题组 ${ }^{[23]}$ 又利用 $\mathrm{CO}$ 作为反应的 $\mathrm{C} 1$ 源, 发展了铜卡宾催化末端脂肪烯烃、 $\mathrm{B}_{2} \mathrm{pin}_{2}$ 和 $\mathrm{CO}$ 的三组 分偶联反应，立体选择性地合成了双硼酸酯取代的环丙 烷类化合物(Eq. 10). 该化合物可经过选择性活化其中 一个 $\mathrm{C}-\mathrm{B}$ 键衍生为单硼酸酯取代的环丙烷, 再经第二 次官能化反应以优秀的产率转化为高附加值的有机分 子. 反应条件温和, 兼容多种官能团, 在有机合成中具 有很高的应用价值，但该反应体系局限于烷基取代的烯 烃.

$$
\begin{aligned}
& \mathrm{R} \curvearrowright+\mathrm{B}_{2} \mathrm{pin}_{2}+\mathrm{CO} \underset{\substack{\mathrm{NaOEt}(1.5 \text { equiv. }) \\
\operatorname{DMAc}(0.4 \mathrm{~mol} / \mathrm{L})}}{\stackrel{\mathrm{IPr} \cdot \mathrm{CuCl}(4 \mathrm{~mol} \%)}{\operatorname{Xantphos}(4 \mathrm{~mol} \%)}} \underbrace{\text { Bpin }}_{\text {Bpin }} \\
& 60^{\circ} \mathrm{C}, 12 \mathrm{~h}
\end{aligned}
$$

作者提出了该环丙烷化反应可能的催化循环机理: 最初 $\mathrm{CuCl}$ 、碱和 $\mathrm{B}_{2} \mathrm{pin}_{2}$ 的相互作用生成活性的 LCuBpin 配合物 $\mathbf{A}$, 反应存在两个催化循环途径, 在其中一个循 环中, $\mathbf{A}$ 与 $\mathrm{CO}$ 配位经插入方式生成 $\mathrm{LCu}(\mathrm{C}=\mathrm{O})$ Bpin 中 间体 $\mathbf{G}$, 接着与 $\mathrm{B}_{2} \mathrm{pin}_{2}$ 反应获得双硼基酮中间体 $\mathbf{H}$; 在
另一循环中，烯烃底物通过配位插入到 $\mathbf{A}$ 的 $\mathrm{Cu}-\mathrm{Bpin}$ 键当中得到烷基铜中间体 $\mathbf{B}$, 随后与另一循环中生成的 酰基硼酸酯 $\mathbf{H}$ 反应获得中间体 $\mathbf{C}$ ，该中间体经分子内重 排转化为中间体 $\mathbf{D}$, 经 1,3-铜迁移后得到中间体 $\mathbf{E}, \mathbf{E}$ 消 除后转化为最终产物环丙烷硼酸酯, 生成 LCuOBpin 配 合物 $\mathbf{F}, \mathbf{F}$ 与 $\mathrm{B}_{2} \mathrm{pin}_{2}$ 反应再生铜配合物 $\mathbf{A}$ 完成该循环过 程(Scheme 8).

\section{9 烯烃的嗍氰基化反应}

2018 年，傅尧课题组 ${ }^{[24]}$ 报道了铜催化试剂控制的 芳基烯烃的区域选择性硼氧基化反应(Scheme 9). 反应 使用结构独特的二甲基丙二腈(DMMN)和 $\mathrm{B}_{2} \mathrm{pin}_{2}$ 作为官 能化试剂, 可以在 $\mathrm{C}=\mathrm{C}$ 双键上同时引入氰基和嗍基, 底物范围广，适用于含不同取代基的芳基烯烃和芳稠环 烯烃. 这种新型的氧基化试剂可以被碱金属离子活化, 获得预期的双官能化产物; 而使用 $N$-氧基- $\mathrm{N}$-苯基对甲 苯磺酰胺(NCTS)作为官能化试剂时，芳基烯烃的邻位 被氰基化，双键被氢硼化，同时在底物分子中引入氧基 和硼基，这与早期 Montgomery 课题组 ${ }^{[25]}$ 及 Buchward 课 题组 ${ }^{[26]}$ 报道的芳基烯烃的嗍氰基化反应结果相一致.

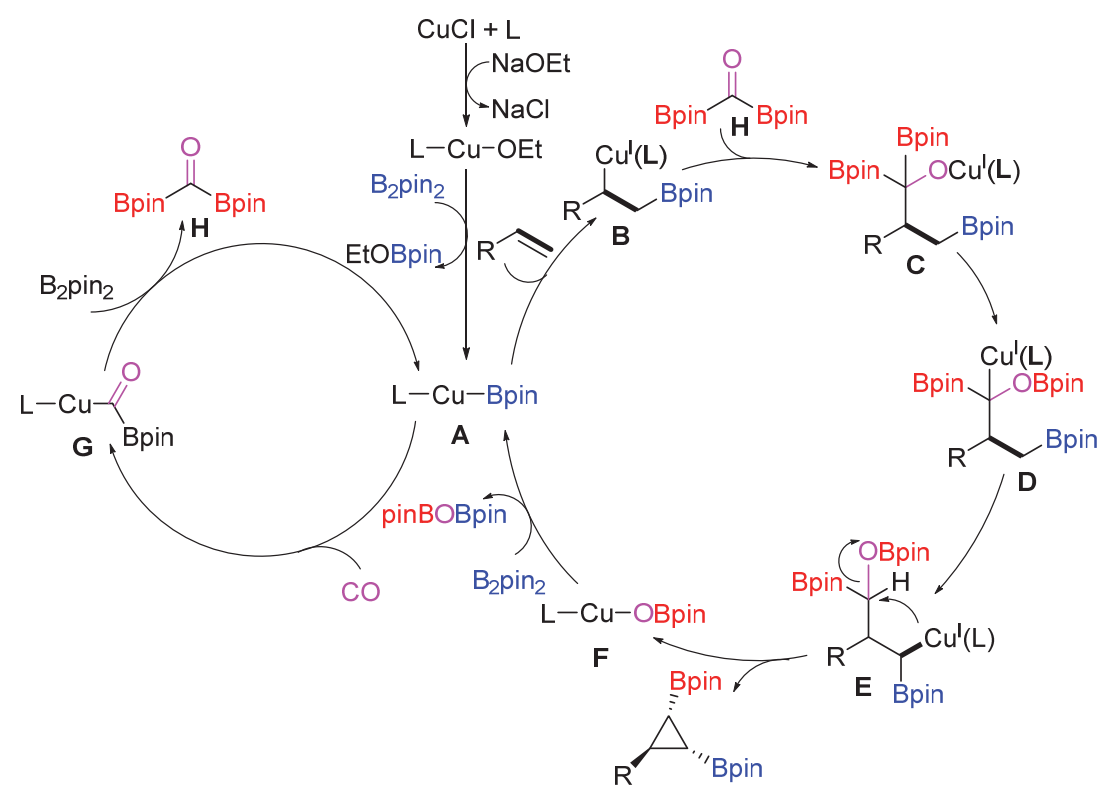

图式 8 铜催化脂肪烯烃的环丙烷化反应可能的机理

Scheme 8 Proposed mechanism of copper-catalyzed cyclopropanation of aliphatic alkenes

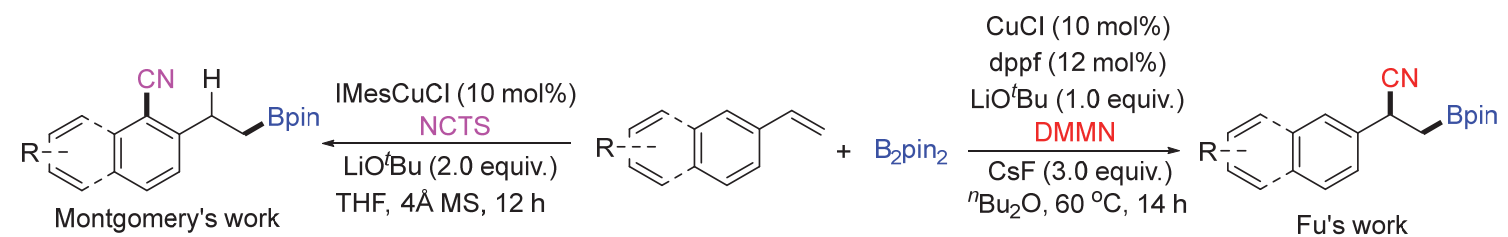

图式 9 铜催化试剂控制的芳基烯烃的区域选择性嗍氰基化反应

Scheme 9 Copper-catalyzed reagent-controlled regioselective cyanoborylation of vinylarenes 


\section{$2 \mathrm{ArB}(\mathrm{OH})_{2}$ 参与的烯烃多组分双官能化反应}

\section{1 烯烃的芳基烷基化反应}

2019 年, 舒伟课题组 ${ }^{[27]}$ 报道了一例铜和光协同催 化下烯烃的自由基接力芳基烷基化反应, 以芳基硼酸作 为芳基化试剂, 以 $\alpha$-溴代羰基化合物为烷基化试剂, 发 展了一种条件温和、实用的方法用于羰基化合物的 $\gamma$ 位芳基化(Eq. 11). 这种接力偶联的策略兼容多种官能 团，在无任何导向基团或预先活化底物的条件下，可以 高效简便地合成 $\gamma$-芳基化的酯、酮、酰胺等羰基化合物.

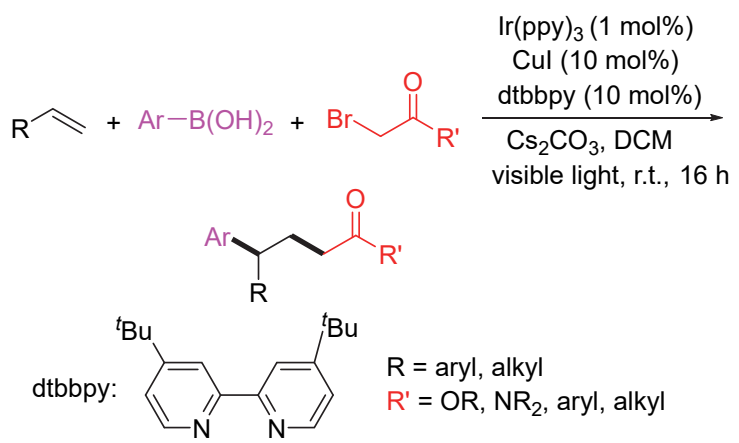

2020 年, 鲍红丽课题组 ${ }^{[28]}$ 开发了一种铜催化下芳 基烯烃、烷基过氧化物和芳基硼酸的三组分反应(Eq. 12). 该方法使用烷基过氧化物作为掩蔽的烷基亲电体, 芳基硼酸作为芳基化试剂, 成功实现了烯烃的烷基芳基 双官能化, 为高附加值 1,1-二芳基烷烃衍生物的合成提 供了新的思路. 反应底物范围广, 官能团兼容性好, 条 件温和, 已经被成功地应用在多种生物活性分子和药物 关键中间体的合成中.

$$
\mathrm{Ar} \curvearrowright+\mathrm{Ar}^{\prime} \mathrm{B}(\mathrm{OH})_{2}+\text { alkyl } \prod_{\mathrm{O}} \mathrm{O}_{\text {alkyl }} \frac{\begin{array}{l}
{\left[\mathrm{Cu}\left(\mathrm{CH}_{3} \mathrm{CN}\right)_{4}\right] \mathrm{PF}_{6}} \\
(5 \mathrm{~mol} \%)
\end{array}}{\begin{array}{l}
\text { Ligand }(7 \mathrm{~mol} \%) \\
\text { DIPEA }(2.0 \text { equiv. }) \\
\text { THF, } \mathrm{N}_{2} \text {, r.t., } 12 \mathrm{~h}
\end{array}}
$$<smiles>[Al]C(C[Te])C[125I]</smiles><smiles>CC(C)C1COC(c2cccc(C3=NC(C(C)C)CO3)n2)=N1</smiles>

最近, 郭丽娜课题组 ${ }^{[29]}$ 使用环烷基硅基过氧化物 和芳基嗍酸为官能化试剂, 实现了 $\mathrm{CuSCN} / 4,4$ '-二溴联 吡啶催化下芳基烯烃的三组分芳基烷基化反应(Eq. 13). 该方法具有温和的反应条件、广泛的底物范围和良好的 官能团兼容性等优点, 为远程羰基官能化的 1,1-二芳基 甲烷衍生物的合成提供了一种有效和直接的途径.

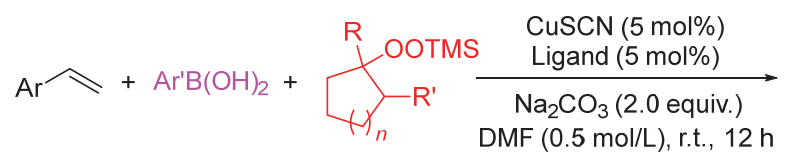<smiles>[R]C(=O)CCCC([R])CC([R])Br</smiles><smiles>Brc1ccnc(-c2cc(Br)ccn2)c1</smiles>

随后, Maruoka 课题组 ${ }^{[30]}$ 使用烷基硅基过氧化物作 为烷基自由基源，芳基嗍酸作为官能化试剂，通过手性 双噁唑和联菜骨架组成的新型配体进行不对称诱导, 实 现了铜催化芳基烯烃的对映选择性芳基烷基化反应(Eq. 14). 该反应底物适用范围广，对映选择性高，条件温 和，为手性 1,1-二芳基烷烃化合物的构建提供了一种新 的策略.

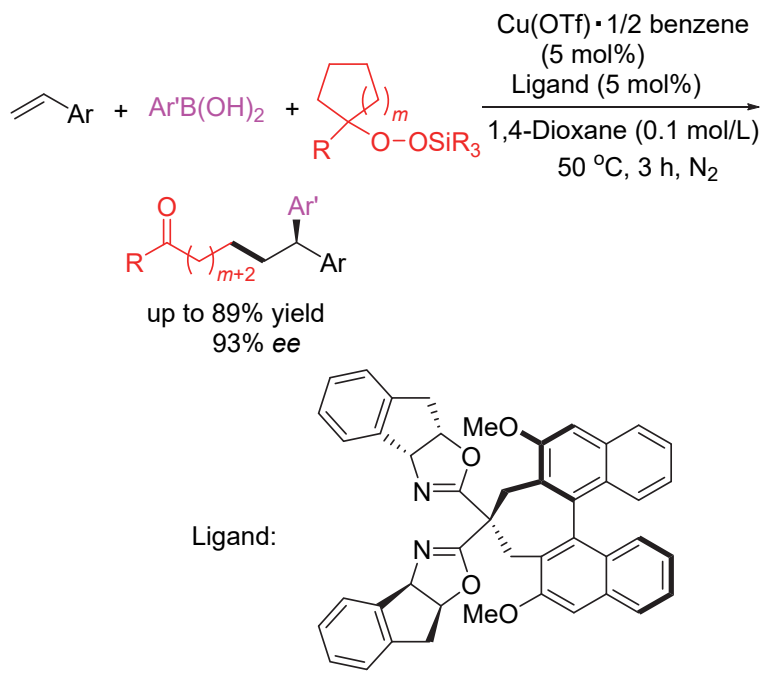

\section{2 烯烃的芳基磺酰化反应}

2020 年, 贾铁争课题组 ${ }^{[31]}$ 报道了 $\mathrm{CuI} / 4,4^{\prime}$-二叔丁基 联吡啶(dtbbpy)催化苯乙烯、硫代苯磺酸甲酯和芳基硼 酸的三组分偶联，通过自由基接力策略实现了烯烃的芳 基磺酰化反应，提供了一种直接高效合成 2,2-二芳基乙 基砜衍生物的新方法(Eq. 15). 该反应成功的关键在于 使用硫代磺酸甲酯作为磺酰基源，在丙酮/ $N, N$-二甲基 甲酰胺(DMF)溶剂体系中成功抑制了反应中生成的磺 酰自由基与芳基硣酸的直接芳基化，转而与苯乙烯底物 进行加成，生成新的茮基自由基，随后捕获体系中的芳 基铜物种 $\mathrm{Ar}^{\prime}\left[\mathrm{Cu}^{\mathrm{II}}\right]$, 经氧化反应生成活性的 $\beta$-磺酰化 $\left[\mathrm{Cu}^{\mathrm{III}}\right]$ 中间体，最终经还原消除提供芳基磺酰化产物， 并再生 $\left[\mathrm{Cu}^{\mathrm{I}}\right]$ 催化剂，催化循环经历了四种不同自由基间 的接力过程(Scheme 10). 该反应可以扩展到不同的烯 烃、硫代磺酸甲酯和芳基硼酸底物，具有高的官能团兼 容性，温和的反应条件非常适合生物活性天然产物的后 
期官能化修饰.

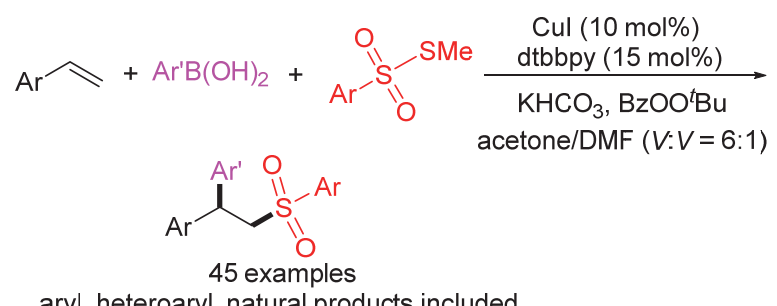

aryl, heteroaryl, natural products included<smiles>CC(C)(C)c1ccnc(-c2cc(C(C)(C)C)ccn2)c1</smiles>

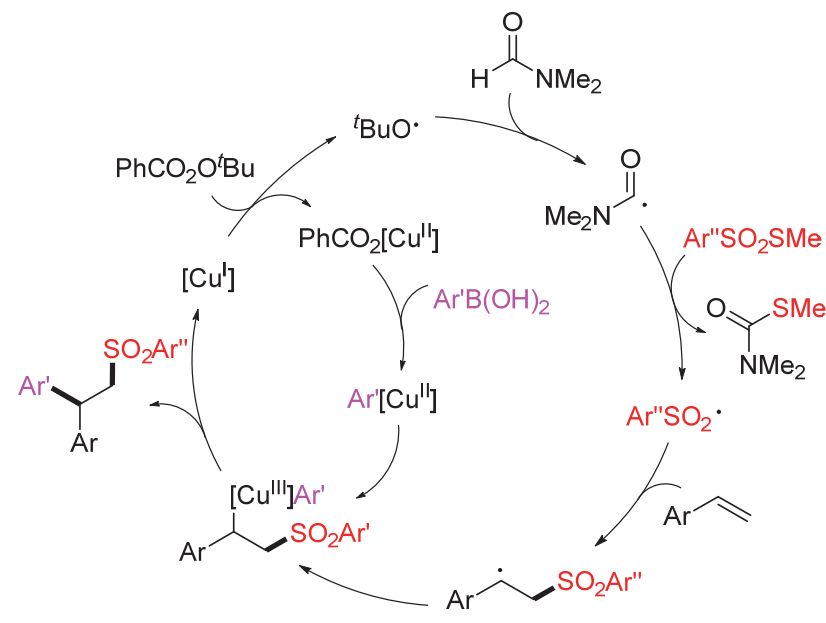

图式 10 铜催化苯乙烯的自由基接力芳基磺酰化反应机理

Scheme 10 Mechanism of copper-catalyzed arylsulfonylation of styrenes via radical relay pathway

\section{$3 \mathrm{RBF}_{3} \mathrm{~K}$ 参与的烯烃多组分双官能化反应}

2020 年, Chemler 课题组 ${ }^{[32]}$ 报道了铜催化芳基烯烃 的三组分碳胺化反应(Eq. 16). 在 $\mathrm{Cu}(\mathrm{OTf})_{2}$ 为催化剂, $\mathrm{MnO}_{2}$ 为氧化剂, 1,10 -菲罗啉(Phen)为配体的条件下, 该 反应以末端或 1,2-二取代烯烃为底物, 烷基三氟硼酸钾 $\left(\mathrm{RBF}_{3} \mathrm{~K}\right.$ )和含不同取代基的脲(或苯胺、酰胺)分别作为官 能化试剂, 在含 $4 \AA$ 分子篮的二氯乙烷中进行, 以中等 到好的产率获得预期的双官能化产物, 为茮基取代的䐂 及胺类衍生物的合成提供了新的途径.

$$
\mathrm{Ar}+\mathrm{R}-\mathrm{BF}_{3} \mathrm{~K}+{ }_{\mathrm{BnHN}} \begin{gathered}
\mathrm{Cu}(\mathrm{OTf})_{2}(10 \mathrm{~mol} \%) \\
\begin{array}{c}
\mathrm{Phen}(12.5 \mathrm{~mol} \%) \\
\mathrm{MnO}_{2}(2.6 \text { equiv. }) \\
\mathrm{DCE}, 105^{\circ} \mathrm{C}
\end{array}
\end{gathered}
$$

\section{4 结论与展望}

从上述反应可以看出, 近三年来铜催化有机嗍试剂
参与的烯烃多组分双官能化反应研究取得了很大的成 就，为分子结构多样性功能有机分子的构建提供了高效 便捷的方法，尤其是 $\mathrm{B}_{2} \mathrm{pin}_{2}$ 参与的反应可以在产物分子 中引入含硼官能团, 有利于产物分子后期的衍生化, 转 化为更有价值的功能性分子，在材料化学和药物化学中 具有重要的应用价值. 尽管如此，该领域仍面临诸多可 能的挑战, 表现在: (1)有机硼试剂的种类有限, 发展新 型硼试剂参与的烯烃多组分双官能化反应仍是一个有 潜力的研究方向; (2)不对称多组分双官能化反应中所用 的手性配体类型相对较少, 主要是手性含磷配体、氮杂 环卡宾和双噁唑骨架; (3)大部分反应的催化剂用量较大 (5 10 mol\%), 需使用毒性较大的有机溶剂, 这些与当 前倡导的绿色合成理念不符, 所以反应体系的开发应该 更加趋向于绿色化学, 降低催化剂用量, 使用绿色溶剂, 实现催化剂的可回收和循环使用等. 我们相信, 随着研 究的不断深入, 有机硼试剂参与的新型烯烃多组分反应 双官能化反应必然会被持续报道出来, 为结构多样性功 能分子的合成提供新的策略, 反应体系也会更加环保高 效. 同时, 有关烯烃多组分双官能化反应机理的深入研 究也将会是该领域的一个重要研究方向.

\section{References}

[1] Wu, Z. X.; Zhang, W. B. Chin. J. Org. Chem. 2017, 37, 2250 (in Chinese).

(吴正兴, 张万斌, 有机化学, 2017, 37, 2250.)

[2] Fu, X. F.; Zhao, W. X. Chin. J. Org. Chem. 2019, 39, 625 (in Chinese). (付晓飞, 赵文献, 有机化学, 2019, 39, 625.)

[3] Zhang, Z.; Gong, L.; Zhou, X. Y.; Yan, S. S.; Li, J.; Yu, D.-G. Acta Chim. Sinica 2019, 77, 783 (in Chinese). (张振, 龚莉, 周晓渝, 颜思顺, 李静, 余达刚, 化学学报, 2019, 77, 783.)

[4] Derosa, J.; Apolinar, O.; Kang, T.; Tran, V. T.; Engle, K. M. Chem. Sci. 2020, 11, 4287.

[5] Badir, S. O.; Molander, G. A. Chem 2020, 6, 1327.

[6] Liu, C.; Zeng, H.; Zhu, C. L.; Jiang, H. F. Chem. Commun. 2020, $56,10442$.

[7] (a) Li, Z.-L.; Fang, G.-C.; Gu, Q.-S.; Liu, X.-Y. Chem. Soc. Rev. 2020, 49,32 .

(b) Dherbassy, Q.; Manna, S.; Talbot, F. J. T.; Prasitwatcharakorn, W.; Perry, G. J. P.; Procter, D. J. Chem. Sci. 2020, 11, 11380.

[8] Itoh, T.; Kanzaki, Y.; Shimizu, Y.; Kanai, M. Angew. Chem., Int. Ed. 2018, 57,8265 .

[9] Lee, J.; Radomkit, S.; Torker, S.; Pozo, J.; Hoveyda, A. H. Nat. Chem. 2018, 10, 99.

[10] Huo, J.; Xue, Y.; Wang, J. Chem. Commun. 2018, 54, 12266.

[11] Kato, K.; Hirano, K.; Miura, M. Chem.-Eur. J. 2018, 24, 5775.

[12] Wu, L.; Zatolochnaya, O.; Qu, B.; Wu, L.; Wells, L.; Kozlowski, M.; Senanayake, C.; Song, J.; Zhang, Y. Org. Lett. 2019, 21, 8952.

[13] Bergmann, A.; Dorn, S.; Smith, K.; Logan, K.; Brown, M. Angew. Chem., Int. Ed. 2019, 58, 1719.

[14] Lee, H.; Lee, S.; Yun, J. ACS Catal. 2020, 10, 2069.

[15] Hu, H.; Hu, X. P.; Liu, Y. H. Angew. Chem., Int. Ed. 2020, 59, 18975.

[16] Butcher, T. W.; McClain, E. J.; Hamilton, T. G.; Perrone, T. M.; Kroner, K. M.; Donohoe, G. C.; Akhmedov, N. G.; Petersen, J. L.; Popp, B. V. Org. Lett. 2016, 18, 6428. 
[17] Lin, S. J.; Lin, Z. Y. Organometallics 2019, 38, 240.

[18] Huang, Y.; Smith, K. B.; Brown, M. K. Angew. Chem., Int. Ed. 2017, 56, 13314.

[19] Fiorito, D.; Liu, Y.; Besnard, C.; Mazet, C. J. Am. Chem. Soc. 2020, $142,623$.

[20] Su, Z.; Feng, Y.; Zou, R.; Qiu, X.; Wang, J.; Tao, C. Chem. Commun. 2020, 56, 7483.

[21] Wu, F.-P.; Yuan, Y.; Schünemann, C.; Kamer, P. C. J.; Wu, X.-F. Angew. Chem., Int. Ed. 2020, 59, 10451.

[22] Yuan, Y.; Wu, F.-P.; Xu, J.-X.; Wu, X.-F. Angew. Chem., Int. Ed. 2020, 59, 17055.

[23] Wu, F.-P.; Luo, X.; Radius, U.; Marder, T. B.; Wu, X.-F. J. Am. Chem. Soc. 2020, 142, 14074.

[24] He, S.-J.; Wang, B.; Lu, X.; Gong, T.-J.; Yang, Y.-N.; Wang, X.-X.;
Wang, Y.; Xiao, B.; Fu, Y. Org. Lett. 2018, 20, 5208.

[25] Zhao, W.; Montgomery, J. Angew. Chem., Int. Ed. 2015, 54, 12683.

[26] Yang, Y.; Buchwald, S. L. Angew. Chem., Int. Ed. 2014, 53, 8677.

[27] Lv, X.; Wang, C.; Wang, Q.; Shu, W. Org. Lett. 2019, 21, 56.

[28] Zhu, X.; Su, M.; Zhang, Q.; Li, Y.; Bao, H. Org. Lett. 2020, 22, 620.

[29] Yang, S.; Gao, P.; Suo, M.-T.; Gao, S.-X.; Duan, X.-H.; Guo, L.-N. Chem. Commun. 2020, 56, 10714.

[30] Sakurai, S.; Matsumoto, A.; Kano, T.; Maruoka, K. J. Am. Chem. Soc. 2020, 142, 19017.

[31] Liang, Q.; Walsh, P. J.; Jia, T. ACS Catal. 2020, 10, 2633.

[32] Kennedy-Ellis, J. J.; Boldt, E. D.; Chemler, S. R. Org. Lett. 2020, 22,8365 .

(Zhao, C.) 